\title{
Repbase Update, a database of repetitive elements in eukaryotic genomes
}

\author{
Weidong Bao ${ }^{1 *}$, Kenji K. Kojima ${ }^{1,2,3^{*}}$ and Oleksiy Kohany ${ }^{1}$
}

\begin{abstract}
Repbase Update (RU) is a database of representative repeat sequences in eukaryotic genomes. Since its first development as a database of human repetitive sequences in 1992, RU has been serving as a well-curated reference database fundamental for almost all eukaryotic genome sequence analyses. Here, we introduce recent updates of RU, focusing on technical issues concerning the submission and updating of Repbase entries and will give short examples of using RU data. RU sincerely invites a broader submission of repeat sequences from the research community.
\end{abstract}

Keywords: Repbase Update, Repbase Reports, Transposable element, RepbaseSubmitter, Database

\section{Background}

Repbase Update (RU), or simply "Repbase" for short, is a database of transposable elements (TEs) and other types of repeats in eukaryotic genomes [1]. Being a wellcurated reference database, RU has been commonly used for eukaryotic genome sequence analyses and in studies concerning the evolution of TEs and their impact on genomes [2-6]. RU was initiated by the late Dr. Jerzy Jurka in the early 1990s and had been developed under his direction until 2014 [7]. Currently, RU continues to be maintained by the Genetic Information Research Institute (GIRI). Free access to RU data is registration-based for academic and non-profit researchers, but a licensing agreement is needed for commercial users. RU and other libraries derived from it are downloaded around 500 times a month from our web server (http://www.girinst.org). In the past 3 years, an average of 159 new users per month have been approved from around the world. As an e-journal accompanying the RU, "Repbase Reports" (RR, ISSN\# 1534-830X) was launched in 2001 to better acknowledge the original contributors to RU entries and to serve as a permanent record. The availability of RU, its data format and implementation, and supplemental tools (Censor and RepbaseSubmitter) were detailed in 2005 and 2006 [1, 8]. This brief paper will focus on recent updates of $\mathrm{RU}$, technical issues concerning the

\footnotetext{
* Correspondence: weidong@girinst.org; kojima@girinst.org

${ }^{1}$ Genetic Information Research Institute, 5150 El Camino Real, Ste B-30, Los Altos, CA 94022, USA

Full list of author information is available at the end of the article
}

submission and updating of Repbase entries, and will give short examples of using RU data.

\section{RU and TE identification}

In eukaryotic genomes, most TEs exist in families of variable sizes, i.e., TEs of one specific family are derived from a common ancestor through its major burst of multiplication in the evolutionary history. A consensus sequence can be reconstructed for each family to approximate the sequence of its ancestral active TEs. Consensus sequences were used to experimentally reconstruct active TEs for transgenesis and insertional mutagenesis [9]. Consensus sequences are especially valuable when classifying TEs and masking repeats, particularly for "old" families of which the sequences have been highly degenerated. The distance from each copy to the consensus is approximately half of the distance between two copies. Family age can be indicated by the average sequence divergence between the consensus and the family members [10].

RU currently contains more than 38,000 sequences of different families or subfamilies, which almost doubled every 3 years since 1999 (Fig. 1). Over $70 \%$ of these entries are complete consensus sequences, unreported elsewhere. The other $30 \%$ of entries represent sample sequences extracted from individual loci (in some cases, the sequences are incomplete). Approximately $90 \%$ of the RU families/subfamilies are collected from a total of 134 species (at least $50 \mathrm{TE}$ families each, Table 1). The remaining $10 \%$ are composed of repeats from another $\sim 700$ species. For the complete list of species and 


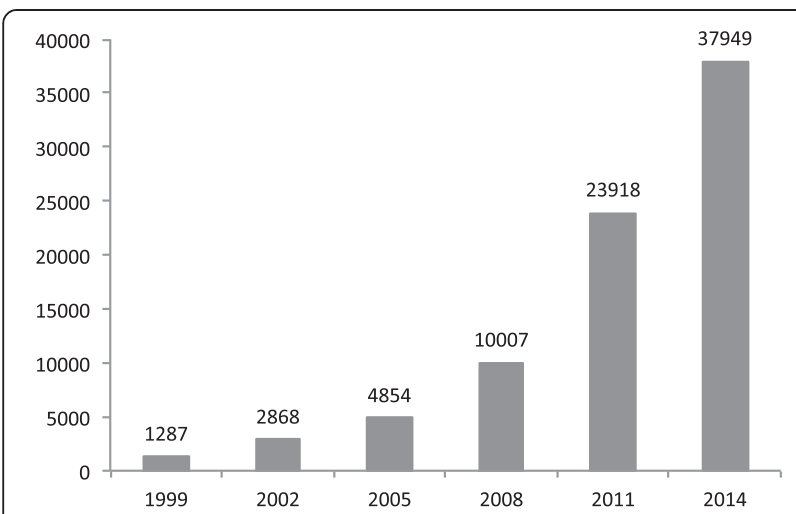

Fig. 1 Numbers of the entries in Repbase Update since 1999

their entry numbers, see Additional file 1. RU also stores non-TE repeat sequences such as satellite sequences, microsatellites, multi-copied RNA genes (rRNA, tRNA, snRNA), and some integrated viral sequences-but these types of sequences are not as thoroughly collected as in other dedicated databases, such as SILVA ribosomal RNA gene database [11], 5S ribosomal RNA database [12], GtRNAdb [13], and "paleovirology" (http://bioinformatics.cvr.ac.uk/paleovirology/).

Exhaustive identification of TEs and the reconstruction of family consensus sequences in complete length are a time-consuming process. Moreover, the challenge of identification varies in different genomes regardless of their size. There are a number of tools designed to automate TE identification and/or annotation, such as RECON [14], RepeatScout [15], PILER [16], RepeatModeler [17], Dfam [18], REPCLASS [19], REPET [20], and
PASTEC [21], with none having distinct advantages [20, 22]. It is noteworthy that these tools use RU as a reference in the classification and annotation process. At GIRI, TE identification mostly involves multiple rounds of running a homemade pipeline based on RECON [14]. A majority-rule consensus sequence is reconstructed from the multiple sequence alignment for each family, and the CpG doublets are optionally compensated for in the consensus, especially in mammalian repeats. About 10-20 sequences are usually sufficient to generate a decent consensus, but fewer sequences can also be used for families of smaller size. In most cases, consensus sequences are manually extended to their real termini, since terminal sequences and TSDs are critical to the classification of TEs (especially non-autonomous ones). For older families, the consensus is often constructed through a two-step process: a pre-build consensus is used to select top-hit sequences and then these sequences are used to build the consensus one more time. Another frequently used tool for TE identification is LTR_FINDER [23], which is used to detect LTR retrotransposons.

\section{TE annotation, classification and naming}

In RU, TEs are currently classified into three groups, i.e., DNA transposons, LTR retrotransposons (including retrovirus), non-LTR retrotransposons (including the SINE category) [24], and further into 65 superfamilies or clades (MuDr, hAT, SINE1, L1 etc.) (Table 2). Conventionally, the term "superfamily" refers to DNA transposons, while the term "clade" applies more frequently to non-LTR retrotransposons and LTR retrotransposons. The classification of autonomous DNA transposons is

Table 1 Top 134 species account for $90 \%$ of the entries

\begin{tabular}{|c|c|c|c|}
\hline Taxonomic group & & No. of species & No. of family \\
\hline \multirow[t]{2}{*}{ Chromalveolata } & Alveolata & 1 & 105 \\
\hline & Oomycetes & 3 & 732 \\
\hline \multirow[t]{11}{*}{ Opisthokonta } & Fungi & 8 & 1595 \\
\hline & Cnidaria & 3 & 1465 \\
\hline & Echinodermata (Strongylocentrotus purpuratus) & 1 & 282 \\
\hline & Hemichordata (Saccoglossus kowalevskii) & 1 & 105 \\
\hline & Lophotrochozoa & 3 & 1122 \\
\hline & Nematoda & 2 & 411 \\
\hline & Arthropoda & 35 & 8161 \\
\hline & Branchiostomidae (Branchiostoma floridae) & 1 & 265 \\
\hline & Tunicata & 2 & 276 \\
\hline & Vertebrata (non-mammalian) & 22 & 9171 \\
\hline & Mammalia & 28 & 4353 \\
\hline \multirow[t]{3}{*}{ Chromalveolata } & Rhodophyta (Chondrus crispus) & 1 & 1181 \\
\hline & Chlorophyceae & 2 & 220 \\
\hline & Embryophyta & 29 & 8101 \\
\hline
\end{tabular}


Table 2 Transposon classification in Repbase

\begin{tabular}{|c|c|}
\hline Group & Superfamily/clade \\
\hline DNA transposon & 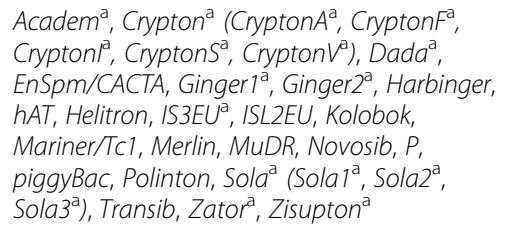 \\
\hline LTR retrotransposon & $\begin{array}{l}\text { BEL, Copia, DIRS, Gypsy, ERV1, ERV2, ERV3, } \\
\text { ERV4 }^{\mathrm{a}} \text {, Lentivirus }\end{array}$ \\
\hline \multirow[t]{2}{*}{ Non-LTR retrotransposon } & $\begin{array}{l}\text { Ambal }{ }^{\text {I }} \text { CR1, CRE, Crack, Daphne, Hero, I, Ingi, } \\
\text { Jockey, Kiri', L1, L2, L2A, L2B, Loa, NeSL, Nimb, } \\
\text { Outcast, Penelope, Proto1, Proto2, R1, R2, R4, } \\
\text { RandI/Dualen, Rex1, RTE, RTETP, RTEX, Tad1, } \\
\text { TX1, Vingi }\end{array}$ \\
\hline & $\begin{array}{l}\text { SINE (SINE1/7SL, SINE2/tRNA, SINE3/5S, } \\
\text { SINE4 }{ }^{\mathrm{a}} \text {, SINEU }{ }^{\mathrm{a}} \text { ) }\end{array}$ \\
\hline
\end{tabular}

${ }^{a}$ Superfamilies/clades added since our latest classification reports [24, 28]

relatively straightforward, often performed by similarity searching of predicted coding sequences, such as by BLASTP or PSI-BLAST. In principal, transposases of one superfamily should not converge with proteins of another when using the PSI-BLAST (an e-value less than 0.005 would be considered converged after several rounds of iterations) $[25,26]$. However, this criterion is overridden if two superfamilies converge separately with different groups of bacterial transposases, such as Zator and Mariner [26], or if peculiar features are found with certain remote groups, such as $\mathrm{Dada}$ and $\mathrm{MuDr}$ [27]. In addition, a superfamily may consist of several distinct subgroups, among which the divergence is insufficient for them to be viewed as distinct superfamilies, such as Sola1, Sola2, and Sola3 in the Sola superfamily [26]. So far, the classification of LTR retrotransposons is also straightforward, but the classification of autonomous non-LTR retrotransposons is largely phylogeny-based instead. For their classification, GIRI provides an online service, called RTclass1 [28], at http://www.girinst.org/ RTphylogeny/RTclass1/. Notably, the classification is subject to ongoing updating once new meaningful data or superfamilies/clades emerge. For example, three previous superfamilies have been recently reclassified into EnSpm (Chapaev, Mirage) and MuDR (Rehavkus) based on weak but significant sequence similarities [24, 29]. Meanwhile, a number of superfamilies were added in recent years-Academ [30], Zisupton [31], and Dada [27] to name but a few. The classification of nonautonomous DNA transposons is largely based on their terminal sequences, TSD features, TIRs, and other structural features (e.g., terminal hairpin in Helitrons). If two non-autonomous DNA TEs have the same TSDs in length and show terminal alignment from position 1 to 11 ( 1 mismatch allowed), they are annotated in the same superfamily in RU.
Each entry in RU, either consensus or sample sequence, represents a "family" or "subfamily" of TEs. Except for a small number of early submitted TEs, the entry name is formatted with the superfamily, subgroup, or clade name, followed by an Arabic number and the species abbreviation [24]. For example, $h A T-4 \_N V$ and $h A T-4 N 1 \_N V$ denote the autonomous family 4 of the hAT superfamily in Nematostella vectensis and the nonautonomous derivative family 1 of the former, respectively [24]. When non-autonomous TEs cannot be clearly classified with present knowledge, they are given general names, such as TE(DNA/LTR/non-LTR)-1_YY, where YY represents its host species. In RU the terms "family" and "subfamily" both correspond to the expanding events of TEs in one specific genome. "Subfamily", however, connotates that two or more closely related TE families were derived from a common ancestral TE. Such subtle difference has more implications for the naming of TEs. Closely related subfamilies usually have similar names differentiated by short modifiers, such as the AluSc or AluSq subfamilies [10], or CR1-3_LMi and CR1-3B_LMi (see below). By contrast, different families are usually assigned with different Arabic numbers. The sequence similarities between retrotransposon families should be less than $80 \%$ if both are consensuses, or less than $70 \%$ if one is a sample sequence, over their whole length or shorter. If the two retrotransposons (consensus sequence or sample sequence) show greater than $80 \%$ identity in more than $50 \%$ of the shorter TE length compared, they are usually considered subfamilies of each other. For DNA transposons, especially Helitron or other long DNA transposons, the above criteria are still applicable in principle, but the similar regions are weighted favorably to their terminal sequences, rather than the other internal sequences, which could be accidentally captured alien sequences. One example of the subfamily naming convention is given by the retrotransposon sequences CR1-3_LMi and CR1-3B_LMi, which are $87 \%$ identical to each other over their entire length. It should be mentioned that their naming does not mean that $C R 1-3 \_L M i$ is a family and CR1-3B_LMi is a subfamily; both are subfamilies if a common ancestral family is implied. To date, not all entries conform to this nomenclature, but they are subject to ongoing updating.

\section{RU updating}

Records in RU are updated regularly. The date of the last update is recorded in each entry for tracking purposes. Updating occurs in different forms: substituting the original sample sequence with a consensus, refining or extending the sequence, adding protein sequences, removing alien (inserted or flanking) sequences, reclassifying, entry renaming, or deletion. The removed older entry versions can be found either in the appendix 
directory of our monthly RU release or in our archived RU releases (http://www.girinst.org/server/archive/). In part, updating is triggered when a batch of new sequences is to be incorporated into RU. Specifically, when the new sequences are compared to all existing sequences in $\mathrm{RU}$, any pair of sequences showing sequence redundancy or name discrepancy will be reexamined. Additionally, some updating comes from candid suggestions by RU users. Credits for the contributors are added in the updated RU entry. To date, more than 5000 entries have been updated at least once.

\section{Submission to RU/RR}

TE sequences can be submitted to the database, RU, or the e-journal, RR. All data published in RR will remain permanently archived and can be quoted like any other article published in a scientific journal. TEs and the accompanying commentaries published in RR are automatically stored in RU and distributed worldwide. Submitting sequences to RU or RR is highly encouraged. Doing so has the potential to increase the visibility of the research paper associated with the deposited sequences, and it should not interfere in any way with the publication of an associated analysis/description of the elements. Besides, the submitter can specify the release date of the submitted TEs by communicating this to GIRI. To date, only 2000 or fewer out of over 38,000 entries have been submitted by RU users outside of GIRI researchers.

Once editorially approved by the editors of RU/RR, the submitted sequences will be released. Any type of sequence, whether consensus or individual sample sequence, is acceptable, even if it is a fragment. However, complete consensus sequences with full annotation are preferred. The primary consideration for inclusion in RU is the novelty of the sequence, which can be checked conveniently at our Censor service (http://girinst.org/ censor/index.php) [8]. In rare cases, if one sequence is nearly identical to any known sequence (for example, around $94 \%$ identity or higher, over its whole length), it should meet one of the following conditions to be accepted to the database: (1) The sequences represent distinct subfamilies, showing at least one significantly divergent region (100-bp or more, insertion/deletion or less than $75 \%$ identity). (2) Each subfamily presents in a large copy number, such as the many nearly identical Alu subfamilies. (3) The two sequences are identified in two remotely related species, where events of horizontal TE transfer are suggested. (4) The submitted sequence is of higher quality (showing intact ORFs, consensus vs. sample sequence) and is intended to replace the older one.

The submission procedure is performed through a Java-based interface called RepbaseSubmitter [8]. It is available for download at http://girinst.org/downloads/ software/RepbaseSubmitter/.
The name (i.e., sequence ID in RU) of the submitted sequence is up to the submitter, but it should be simple and informative. By selecting the "Auto" tab on the "Summary" page during submission, RepbaseSubmitter will generate a unique name for the sequence based on its classification. The automatically generated name can then be modified to indicate whether the sequence represents a subfamily or a non-autonomous $\mathrm{TE}$. In the "Reference" page of RepbaseSubmitter, users may be confused between the options to choose "Direct Submission to Repbase Update" or "Direct Submission to RR" in the "Submission" > "Select Repository" pull-down menu. The "RR" stands for "Repbase Reports". If the sequence is a consensus and unreported elsewhere, RR is more suitable. Notably, RepbaseSubmitter does support batch submission by selecting the "Submit All" tab. However, this requires that all sequences be correctly IG-formatted, and each has a unique name. For this purpose, users can choose to save the RepbaseSubmitterprocessed, properly formatted individual sequences into one file for the batch submission.

\section{Using RU}

The monthly release of RU is available in both FASTA and EMBL formats. Only the EMBL files contain full annotations, such as TE classification, host species, release version, release date, latest update date, references, and comments. The EMBL files can be transformed into a relational database for local use. To detect repeat sequences in the genome sequences, FASTA-formatted RU data can be directly used with the standard homology search tools, such as BLAST programs, cross_match, and Censor [8]. Other TE annotation tools, such as RepeatMasker [32] and REPET [20], may need RU data in different formats. These toolspecific variants of RU can be downloaded from GIRI's website, but they are prepared by the authors of the tools and are not updated on a monthly basis. The TE library used by RepeatMasker is essentially identical to the RU dataset, except for the format and the accompanying annotation-supportive files. However, it may show some minor sequence differences to RU at times for various reasons (see the README file in its package, downloadable at GIRI website http://girinst.org/server/RepBase/index.php). Various pre-masked genome sequences generated by RepeatMasker are available at the UCSC genome browser website (https://genome.ucsc.edu). In addition to RepeatMasker, RU is also essential for the Dfam database [18], where the profile hidden Markov models (profile HMMs) for different repeats are used in conjunction with the HMM search tool nhmmer to detect repetitive sequences in the genome [18]. Dfam is unique in that it does not rely on a homology-based search tools, but building profile HMMs is still dependent on the quality of the consensus sequences deposited in RU. 
Depending on the specific aim, in many cases, only a subset of RU is needed. One can conveniently extract essential information by working in UNIX/Linux system. For example the AWK command, "awk 'BEGIN $\{F S=$ "//"; RS = "\0"; ORS = "//"\}\{for (i = 1; i <=NF; $\mathrm{i}++)$ if (\$i $\sim / n K W . " h A T ; / \& \& \$ i \sim / 7$-bp TSD/) print \$i\}' XXX.ref", will extract all those hAT families annotated with "7-bp TSD" from the EMBL file "XXX.ref". With minor modifications, this command can also be used for extracting entries from a specific species or taxonomic group. Alternatively, on GIRI's website, users can perform basic text searches at http://girinst.org/repbase/update/ search.php, or search and download entries with specific taxonomic names or repeat classes at http://girinst.org/ repbase/update/browse.php.

\section{Conclusions}

For years, RU has been serving as a well-curated repeat library in virtually all eukaryotic genome research. At present, most entries in RU were submitted by researchers at GIRI and are not reported anywhere else besides RR. On the other hand, we highly encourage outside researchers to submit their repeat sequences to either RR or RU to expand the current repository of TEs thereby benefiting the whole research community. Meanwhile, RU will make every effort to keep up with the pace of newly sequenced genomes without sacrificing the established quality standards. Priority is placed on new genomes that are taxonomically less represented in RU. Suggestions for genomes to be analyzed are welcome. Until now, in each monthly release, RU entries are divided into several files according to the taxonomic origin. A number of entries may appear in multiple files (http://girinst.org/repbase/ update/index.html). The separating of entries into individual files is becoming increasingly unnecessary, especially when the genomes analyzed are getting more diverse than before. For this reason, future releases of RU may be prepared as an all-in-one file, together with instructions and scripts to extracting the target groups. Another planned new feature of RU is a "Reference" protein library for each TE superfamily, which will comprise high-quality proteins only. This will be accomplished by using only recently active families. To avoid uncertainty derived from consensus building and/or exon-intron prediction, transposases will be selected from those without intron or with mRNA evidence. This protein set would be useful in understanding the diversity of TE-encoded proteins and their impact on the evolution of host genomes.

\section{Availability and requirements}

- Project name: Repbase Update

- Project home page: http://www.girinst.org/repbase/ update/index.html
- Operating system(s): Any system

- Programming language: N/A

- Other requirements: N/A

- License: a custom user agreement for RU

- Any restrictions to use by non-academics: license needed

\section{Additional file}

Additional file 1: Species and the number of TE family in RU. Data is up to the end of 2014.

\section{Abbreviations}

GIRI: Genetic Information Research Institute; LTR: Iong terminal repeat; ORF: open reading frame; RU: Repbase Update; RR: Repbase Reports; TE: transposable element; TIR: terminal inverted repeat; TSD: target site duplication.

\section{Competing interests}

The authors declare that they have no competing interests.

\section{Authors' contributions}

WB and KKK performed research and drafted the manuscript. OK designed the software Censor and RepbaseSubmitter. All authors edited and approved the manuscript.

\section{Acknowledgments}

This manuscript is dedicated to the memory of our friend and colleague, Jerzy Jurka, who passed away on July 19th, 2014. The authors would like to thank the reviewer for many valuable comments and Karolina Walichiewicz for the help with editing the manuscript.

\section{Author details}

${ }^{1}$ Genetic Information Research Institute, 5150 El Camino Real, Ste B-30, Los Altos, CA 94022, USA. ²Department of Computational Biology and Medical Sciences, Graduate School of Frontier Sciences, University of Tokyo,

Minato-ku, Tokyo, Japan. ${ }^{3}$ Institute of Medical Science, University of Tokyo, 4-6-1 Shirokanedai Minato-ku, Tokyo 108-8639, Japan.

Received: 24 February 2015 Accepted: 17 April 2015

Published online: 02 June 2015

\section{References}

1. Jurka J, Kapitonov W, Pavlicek A, Klonowski P, Kohany O, Walichiewicz J. Repbase Update, a database of eukaryotic repetitive elements. Cytogenet Genome Res. 2005;110:462-7.

2. Huda A, Marino-Ramirez L, Jordan IK. Epigenetic histone modifications of human transposable elements: genome defense versus exaptation. Mob DNA. 2010;1:2.

3. Han MJ, Xu HE, Zhang HH, Feschotte C, Zhang Z. Spy: a new group of eukaryotic DNA transposons without target site duplications. Genome Biol Evol. 2014;6:1748-57.

4. Pace JK, Gilbert C, Clark MS, Feschotte C. Repeated horizontal transfer of a DNA transposon in mammals and other tetrapods. Proc Natl Acad Sci U S A. 2008;105:17023-8.

5. Suh A, Churakov G, Ramakodi MP, Platt RN, Jurka J, Kojima KK, et al. Multiple lineages of ancient CR1 retroposons shaped the early genome evolution of amniotes. Genome Biol Evol. 2014;7:205-17.

6. Wallau GL, Ortiz MF, Loreto EL. Horizontal transposon transfer in eukarya: detection, bias, and perspectives. Genome Biol Evol. 2012;4:689-99.

7. Jurka J, Walichiewicz J, Milosavljevic A. Prototypic sequences for human repetitive DNA. J Mol Evol. 1992;35:286-91.

8. Kohany O, Gentles AJ, Hankus L, Jurka J. Annotation, submission and screening of repetitive elements in Repbase: RepbaseSubmitter and Censor. BMC Bioinformatics. 2006;7:474.

9. Ivics Z, Hackett PB, Plasterk RH, Izsvak Z. Molecular reconstruction of Sleeping Beauty, a Tc1-like transposon from fish, and its transposition in human cells. Cell. 1997;91:501-10. 
10. Kapitonov V, Jurka J. The age of Alu subfamilies. J Mol Evol. 1996;42:59-65.

11. Quast C, Pruesse E, Yilmaz P, Gerken J, Schweer T, Yarza P, et al. The SILVA ribosomal RNA gene database project: improved data processing and web-based tools. Nucleic Acids Res. 2013;41:D590-6.

12. Szymanski M, Barciszewska MZ, Barciszewski J, Erdmann VA. 5 S ribosomal RNA database Y2K. Nucleic Acids Res. 2000;28:166-7.

13. Chan PP, Lowe TM. GtRNAdb: a database of transfer RNA genes detected in genomic sequence. Nucleic Acids Res. 2009:37:D93-7.

14. Bao Z, Eddy SR. Automated de novo identification of repeat sequence families in sequenced genomes. Genome Res. 2002;12:1269-76.

15. Price AL, Jones NC, Pevzner PA. De novo identification of repeat families in large genomes. Bioinformatics. 2005;21 Suppl 1:i351-8.

16. Edgar RC, Myers EW. PILER: identification and classification of genomic repeats. Bioinformatics. 2005;21 Suppl 1:1152-8.

17. Smit AFA, Hubley R. RepeatModeler Open-1.0. 2008-2015. (http://www.repeatmasker.org).

18. Wheeler TJ, Clements J, Eddy SR, Hubley R, Jones TA, Jurka J, et al. Dfam: a database of repetitive DNA based on profile hidden Markov models. Nucleic Acids Res. 2013:41:D70-82.

19. Feschotte C, Keswani U, Ranganathan N, Guibotsy ML, Levine D. Exploring repetitive DNA landscapes using REPCLASS, a tool that automates the classification of transposable elements in eukaryotic genomes. Genome Biol Evol. 2009;1:205-20.

20. Flutre T, Duprat E, Feuillet C, Quesneville $H$. Considering transposable element diversification in de novo annotation approaches. PLoS One. 2011;6:e16526.

21. Hoede C, Arnoux S, Moisset M, Chaumier T, Inizan O, Jamilloux V, et al. PASTEC: an automatic transposable element classification tool. PLoS One. 2014;9:e91929.

22. Saha S, Bridges S, Magbanua ZV, Peterson DG. Empirical comparison of ab initio repeat finding programs. Nucleic Acids Res. 2008;36:2284-94.

23. $\mathrm{Xu} Z$, Wang H. LTR_FINDER: an efficient tool for the prediction of full-length LTR retrotransposons. Nucleic Acids Res. 2007;35:W265-8.

24. Kapitonov W, Jurka J. A universal classification of eukaryotic transposable elements implemented in Repbase. Nat Rev Genet. 2008;9:411-2. author reply 414 .

25. Kapitonov W, Jurka J. RAG1 core and V(D)J recombination signal sequences were derived from Transib transposons. PLoS Biol. 2005;3:e181.

26. Bao W, Jurka MG, Kapitonov W, Jurka J. New superfamilies of eukaryotic DNA transposons and their internal divisions. Mol Biol Evol. 2009;26:983-93.

27. Kojima KK, Jurka J. A superfamily of DNA transposons targeting multicopy small RNA genes. PLoS One. 2013;8:e68260.

28. Kapitonov W, Tempel S, Jurka J. Simple and fast classification of non-LTR retrotransposons based on phylogeny of their RT domain protein sequences. Gene. 2009;448:207-13.

29. Yuan YW, Wessler SR. The catalytic domain of all eukaryotic cut-and-paste transposase superfamilies. Proc Natl Acad Sci U S A. 2011;108:7884-9.

30. Kapitonov W, Jurka J. Academ - a novel superfamily of eukaryotic DNA transposons. Repbase Reports. 2010;10:643.

31. Bohne A, Zhou Q, Darras A, Schmidt C, Schartl M, Galiana-Arnoux D, et al. Zisupton-a novel superfamily of DNA transposable elements recently active in fish. Mol Biol Evol. 2012;29:631-45.

32. Smit AFA, Hubley R, Green P. RepeatMasker Open-3.0. 1996-2010 (http://www.repeatmasker.org).

\section{Submit your next manuscript to BioMed Central and take full advantage of:}

- Convenient online submission

- Thorough peer review

- No space constraints or color figure charges

- Immediate publication on acceptance

- Inclusion in PubMed, CAS, Scopus and Google Scholar

- Research which is freely available for redistribution 